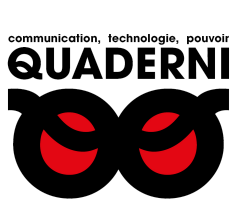

\title{
Quaderni
}

Communication, technologies, pouvoir

96 | Printemps 2018

Smart city : "fiction" et innovation stratégique

\section{La Ville intelligente : objet au cœur de nombreuses controverses}

\section{Emmanuel Eveno}

\section{(2) OpenEdition}

1 Journals

\section{Édition électronique}

URL : https://journals.openedition.org/quaderni/1174

DOI : 10.4000/quaderni. 1174

ISSN : 2105-2956

Éditeur

Les éditions de la Maison des sciences de l'Homme

\section{Édition imprimée}

Date de publication : 15 mai 2018

Pagination : $29-41$

\section{Référence électronique}

Emmanuel Eveno, "La Ville intelligente : objet au cœur de nombreuses controverses », Quaderni [En ligne], 96 | Printemps 2018, mis en ligne le 15 mai 2020, consulté le 28 septembre 2021. URL : http:// journals.openedition.org/quaderni/1174; DOI : https://doi.org/10.4000/quaderni.1174 


\section{$D$ ossier}

\section{La Ville intelligente : \\ objet au cœur \\ de nombreuses \\ controverses}

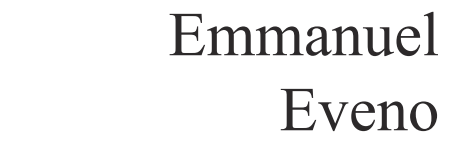

Professeur de géographie Directeur du LISST-Cieu
Le succès à la fois récent et manifeste de l'expression «Smart City/Ville intelligente » soulève de nombreuses questions. Elle est au cœur de nombreux débats et controverses, que ce soit au sein du monde des acteurs de la ville ou dans celui des chercheurs en sciences sociales. Le succès de l'expression auprès des uns la rend d'autant plus suspecte aux yeux des seconds.

C'est que, à la différence de nombreux concepts qui se sont imposés dans les politiques publiques au cours de dernières décennies, en particulier celui de " développement durable » ou celui de « société de l'information», celui de « Ville intelligente $^{1} »$ ne semblait rien devoir, jusqu'à il y a peu, aux mondes académiques tandis qu'il était souvent perçu comme ayant été imposé par des acteurs économiques en quête de nouveaux marchés. Dans le registre de la légitime circulation des idées et des concepts entre les mondes politiques et scientifiques, la «Ville intelligente» serait en quelque sorte une intruse, un objet sans fondement, sans épaisseur, sans problématisation véritable.

\section{L'engouement vis-à-vis des Villes intelligentes}

Le problème tient aussi au fait que l'expression, si elle ne fait pas consensus, a toutefois été maintes fois reprise, réappropriée et ceci dans des sens et par des acteurs très variés. On trouve aujourd'hui un grand nombre de «Villes intelligentes » ou de villes qui se revendiquent comme telles dans le monde.

Par le recours très fréquent à l'expression en langue anglaise «Smart City » plutôt qu'à sa traduction (approximative, il faut bien le re- 
connaître) en " Ville intelligente ", les acteurs qui s'en emparent semblent en effet céder à des stratégies de communication de type incantatoire. Il s'agit de se rendre attractifs et, pour cela, la langue anglaise conjuguée aux outils de la modernité technologique comme au monde des affaires semble s'imposer.

Il s'agit là d'un phénomène bien connu, la circulation des modèles en termes de développement territorial se fait souvent sur le principe du mimétisme. C'est ainsi que le développement des zones dédiées à l'innovation économique en France a vu l'éclosion de ce que Raymond Woessner a appelé « un nouvel archétype spatial ${ }^{2}$ », se signalant par l'emploi du mot «Valley» : «Cosmetic Valley», "Aerospace Valley», "Telecom Valley", «Silver Valley», «Optic Valley», «TIC Valley», «Mechanic Valley »... etc. (on ne parle ici, bizarrement, que d'exemples situés en France). Ils sont devenus les toponymes marqueurs de ces ambitions politiques. Il s'agit en fait d'invoquer la «Silicon Valley », modèle indépassé de la technopole et du développement des technologies numériques («digitales » diraient certains amateurs d'anglicismes).

À bien des égards, la « Smart city» reprend ce même schéma et importe, sans beaucoup de précautions parfois, des dispositifs ou des concepts nés pour beaucoup d'entre eux aux États-Unis. Au toponyme «Valley » se rajoute, comme en nouveau viatique de la modernité urbaine, le mot «Lab» (pour «Laboratory»). L'inspiration vient alors plutôt de la côte Est des États-Unis, très spécifiquement du Massachussetts Institut of Technology (M.I.T.). C'est ainsi que la « Smart City » est très souvent reliée aux «Living labs » et autres «Fab labs», inventés l'un et l'autre au M.I.T. et que, bien souvent, au cœur de l'organigramme des métropoles qui se revendiquent «Smart Cities », on trouve un «Urban Lab». Ces «Labs» témoignent de l'évolution de la fabrique urbaine et de sa régulation vers la prise en compte de méthode qui reposent sur l'expérimentation et sur la mobilisation d'usagers-testeurs.

Au-delà du succès des mots, le Sommet Habitat III qui s'est tenu à Quito en octobre 2016, a été un temps fort de l'affichage par les acteurs de la ville de leurs intérêts à comprendre et se situer par rapport à ce que pouvaient être ces « Villes intelligentes $»$. Le nombre très significatif d'événements parallèles organisés sur ces questions à l'occasion de ce Sommet a en effet permis d'apprécier que les acteurs urbains ont sensiblement adhéré aux idées selon lesquelles les «Villes intelligentes 》 représentaient bien une version possible et crédible de la ville du futur.

«Accueillir un événement smart cities devient un enjeu en soit, outre les retombées économiques directes attendues en matière d'hébergement et de restauration. Et la concurrence devient rude entre villes ${ }^{3} »$ affirmait Guillaume Bermond pour le journal Le Monde. Le site officiel de la ville de Bruxelles a recensé un ensemble de 26 événements internationaux de types salons ou congrès qui porteront sur la « Ville intelligente » dans le courant de l'année 2018. Cette liste, déjà très significative, est toutefois incomplète, elle laisse de côté toute une série d'autres événements de portées sans doute plus réduite... mais qui ne sont pour autant pas sans ambition, comme le «Smart City Expo » qui se tiendra à Casablanca en avril 2018, ou encore l'événement « Smart Cities » 
régulièrement organisé à Sofia, en Bulgarie, depuis 2008, le « Smart City Forum » de Varsovie, en Pologne, qui en sera à sa cinquième édition annuelle consécutive, ou encore les "Forum Smart City » organisé depuis trois ans par le périodique économique La Tribune en partenariat avec sept métropoles françaises : Paris, Nice, Marseille, Montpellier, Toulouse, Bordeaux, Nantes.

\section{Les classements internationaux}

Les classements internationaux sont un autre élément d'explication de la diffusion rapide de l'expression «Ville intelligente ». L'un des classements parmi les plus anciens est celui fourni depuis 2002 par l'« Intelligent Community Forum ». Il repose sur la candidature des villes qui doivent remplir un formulaire sur le site. Dans un premier temps, 21 d'entre-elles sont retenues, puis 7. Depuis 2002, la géographie de ces nominations est tout à fait intéressante. Si on retient le nombre de villes différentes par pays (plusieurs villes se sont présentées sur plusieurs années), il faut alors admettre que les pays dans lesquelles ces « Villes intelligentes » se développent prioritairement sont le Canada, les États-Unis et Taiwan, puis, loin derrière mais de façon équilibrée, le Royaume-Uni, la Corée du Sud, le Japon et l'Australie. Tous les autres pays (dont la France) n'apparaissent qu'à travers une seule ville.

Du fait même que la méthode repose sur la candidature spontanée des villes et qu'elle semble de fait mieux adaptée au monde anglophone qu'au reste du monde (il faudrait alors effectivement parler de «Smart Cities »), ces palmarès sont évidemment contestables. On n'y trouve par exemple aucune ville d'Amérique latine, aucune d'Afrique (ce qui est moins surprenant) et pas davantage quelques villes par ailleurs très reconnues comme des modèles du genre. Par exemple, on n'y trouve pas Barcelone, on n'y trouve Singapour que pour les Palmarès des années 2002 et 2004 ...

Depuis 2015, Boyd Cohen, directeur de l'innovation et professeur à la Universidad del Desarrollo de Santiago du Chili produit un classement particulièrement raffiné des "Villes intelligentes » dans le monde en considérant une très large palette de critères. L'intérêt de son approche c'est qu'elle ne repose pas sur les déclarations de candidature des villes. Sur 120 villes considérées comme éligibles sur l'ensemble du monde, il en retient finalement 30 par grandes régions du monde : l'Europe, l'Amérique du Nord, l'Amérique latine et l'Asie-Pacifique. Au-delà de la robustesse apparente de sa méthodologie, il a acquis une forte notoriété auprès des villes tentées par les retombées d'un tel classement en raison du sérieux de la démarche, qui s'appuie sur un Conseil scientifique dans lequel figurent un représentant d'I.B.M., ainsi que plusieurs représentants directs ou indirects de la « Smart City Barcelona » mais aussi en raison du « design » de sa méthodologie, réifiée par la «Smart City Wheel », soit une sorte de figure à partir de laquelle les villes peuvent se représenter assez aisément leur position.

En fait, les collectivités se réfèrent assez normalement aux méthodes qui les avantagent et l'essentiel de celles qui nourrissent quelques ambitions sur la problématique des «Villes 
intelligentes » peuvent aisément trouver une possibilité de visibilisation médiatique. Par exemple, en 2013, soit au début de son mandat, le maire de Montréal s'était engagé personnellement à ce que sa ville devienne une ville intelligente et soit reconnue comme telle. Cette ambition politique s'est trouvée consacrée en 2017 lorsque Montréal a reçu le prix Smart Cities Award 2017 de la Fédération Internationale de l'Automobile (FIA) dans le cadre du Forum Smart Cities, organisé à l'occasion de la première course de Formule E dans la métropole.

\section{Des villes intelligentes partout?}

Si on tente de cerner les contours des projets français qui s'imposent avec le plus d'évidence, ceux qui sont cités en exemple dans les médias, dans les documentations officielles ainsi que dans les différents travaux scientifiques, ceux qui fournissent une abondante argumentation pour se revendiquer de l'expression « Ville intelligente »... il reste bien difficile de discerner les traits communs entre eux. La comparaison de ce qui se passe dans les quelques villes concernées : Lyon, Paris, Nice, Mulhouse, Besançon, Issy-Les-Moulineaux, Dijon, Lille, Toulouse, Nantes, Bordeaux, Montpellier... laisse apparaître de grosses différences, que ce soit en termes de conception, de méthode, de mises en œuvre, d'ambitions ou de réalisations. Force est de constater que les projets se dessinent en avançant, à l'épreuve des faits et dans le dialogue avec d'autres.

La difficulté à s'en tenir à une définition claire est encore accrue lorsqu'il s'agit d'aborder les initiatives éparses, en dehors de toute formalisation ou de tout affichage d'une ambition politique. Dans de nombreux cas, le recours à une technologie dite « intelligente » semble valoir légitimité à faire partie de ce type de ville.

À étendre l'expression de «Ville intelligente » à ces cas, on court le risque de la vider de son sens. Or, devant le succès médiatique de l'expression, le risque est réel. Il suffit pour s'en convaincre de mettre en regard la courte liste que nous venons d'évoquer et les résultats présentés par l'enquête réalisée par IDC pour le Synthec numérique et qui portait sur 102 collectivités locales (communes de plus de 5000 habitants, communautés urbaines et communautés d'agglomérations). Cette enquête révèle en effet «que $93 \%$ des communes interrogées "ont déjà lancé une initiative de ville intelligente" mais elles s'inscrivent dans un périmètre large de smart city. ${ }^{4}$ "

Ce constat d'une tendance apparente à la diffusion tous azimuts de la «Ville intelligente » est corroboré par l'enquête réalisée par La Gazette des communes et m2oCity entre le 23 mars et le 13 avril 2017 : « La ville intelligente aurait-elle dépassé le stade des pionniers? Révélant qu'une collectivité sur deux a entamé une réflexion sur la smart city, une étude La Gazette - m2oCity (...) permet de le penser. Près de la moitié des répondants ont élaboré une stratégie de ville intelligente ou comptent le faire, même si $15 \%$ seulement ont engagé des projets concrets. Des chiffres qui pourraient présager une large progression des projets à court terme, estiment les auteurs de l'étude 5 . » L'enquête révèle en outre que la « Ville intelligente » pourrait même exister sans en passer par un projet politique, qu'elle pourrait exister sans même que les acteurs de la ville le revendiquent, voire en aient conscience : 
«à l'instar de Monsieur Jourdain qui faisait de la prose sans le savoir, des collectivités pratiquent déjà des démarches "smart city" : $48 \%$ des collectivités qui n'ont pas encore de stratégie, mais comptent en adopter une, et $24 \%$ de celles qui ne l'envisagent pas utilisent des objets connectés : eau, énergie, capteurs de pollution.»

Ces enquêtes laissent penser que le phénomène des « Villes intelligentes » pourrait être à la portée de tous types de collectivités. L'Observatoire Smart City lancé par la société Tactis en 2015 a ainsi considéré qu'il fallait élargir son champ d'observation: «d'abord centré sur les politiques déployées par les villes et les agglomérations, l'Observatoire a élargi en 2016 son champ d'analyse aux territoires, en incluant les projets portés par les structures intercommunales (syndicats mixtes, groupements de communes, entreprises locales de type SEM, SPL, SICAE.... . ${ }^{6}{ }^{\prime}$

On retrouve là un schéma d'argumentation qui va au-devant des attentes de la population des élus locaux qui sont à l'écoute des évolutions sociétales et à l'affut de nouveaux modèles de développement, de signes de la modernité. Ce schéma est notoirement alimenté par les acteurs économiques, soucieux d'augmenter la taille de leur marché potentiel. C'est ce même schéma qui avait prévalu au moment où le concept de « technopole » était diffusé en France, soit à partir des années 1980. Initialement considérées comme indissociables d'un campus universitaire et d'un milieu industriel à la fois dynamique et fortement investies dans la Recherche \& Développement, les technopoles étaient a priori vouées à essaimer dans les principales villes universitaires et industrielles. Or, le concept a eu un tel succès qu'il s'est peu à peu dilué en diffusant dans des territoires où ces acteurs-clés étaient absents ou quasi absents. Au début de la décennie 1990, la DATAR avait réalisé un atlas ${ }^{7}$ des technopoles dans le monde, dans lequel, chose très étonnante, on constatait que la France en avait un nombre très supérieur à n'importe quel autre pays au monde.

\section{Interpréter ces émergences : les limites de l'approche littéraliste}

Il apparaît en fait de plus en plus nécessaire, si on veut se confronter aux questions posées autour de cette émergence récente, de faire un tri entre les différentes directions et les différentes options qu'incarne l'expression "Ville intelligente ». Certains auteurs, dont Antoine Picon, proposent de prendre l'expression dans son sens littéral: « Ce livre part d'une hypothèse en apparence simple: dans la smart city, dans la ville intelligente, il convient de prendre le terme intelligent en un sens beaucoup plus littéral qu'il pourrait y paraître. Intelligent au sens de ce qui apprend, comprend, raisonne. ${ }^{8} \gg$ Cette approche présente toutefois, selon nous, le danger d'une réduction métaphorique. Attribuer de l'intelligence à la ville reviendrait à la considérer comme un être humain, au moins comme un animal, en tout cas un être vivant. L'hypothèse formulée par Antoine Picon ne tombe pas dans ce piège puisqu'il propose de déplacer la question de l'intelligence vers des entités non-humaines, vers l'intelligence artificielle. Ceci étant, de nombreuses références à la « Ville intelligente » n'ont pas cette subtilité. C'est ainsi que les variations qui s'opèrent autour de cette expression nous informent sur ce piège du littéralisme. Par exemple, l'une des contestations parmi les plus courantes des «Villes intel- 
ligentes » consiste à opposer un mot à un autre, l'antonyme en l'occurrence : s'il y a des « Villes intelligentes », ce serait donc qu'il y a aussi des villes ou des villages $«$ stupides $»$.

Autre contestation encore, qui tend à distendre le sens d'une expression en mal de définition, celle qui promeut le transfert de la notion de ville vers celle de « territoire » et qui, de ce fait, porte la revendication des acteurs des territoires non-urbains à ne pas se laisser enfermer dans cette affreuse distinction entre intelligence et stupidité. Ce n'est certes pas le fond du propos développé par le Guide réalisé en France par la Caisse des Dépôts « Smart City versus Stupid Village », qui se soucie d'ailleurs assez peu de ce que serait ou ne serait pas une «Smart City» pour se consacrer à la promotion de nouveaux outils en lien avec la nécessaire modernisation des collectivités locales. En allant au-devant des desiderata des élus locaux des territoires des villes petites et moyennes, la Caisse se fait l'écho d'une préoccupation constante de l'aménagement du territoire : "Le concept de smart city est-il uniquement réservé aux métropoles ou aux grandes villes? Les plus petits territoires ne peuvent-ils pas, eux aussi, prendre part à ce mouvement? 's'interrogeait ainsi Cédric Verpeaux, l'un des auteurs de ce Guide ${ }^{10}$. On pourrait utilement se demander si le modèle des «Villes intelligentes » n'est pas en contradiction forte avec cette « passion française ${ }^{11}$ de l'égalité des territoires ? Il faudra alors faire la distinction entre « Ville intelligente » et modernisation des collectivités locales.

Cette conception littérale de la « Ville intelligente » se retrouve assez naturellement dans l'ensemble des démarches d'opérationnalisation. Elle se transforme alors en argument pour décider les acteurs d'une ville ou d'un territoire à entamer une politique allant dans le sens de la transformation de leur ville ou territoire en entité « intelligente ». C'est ce que l'on constate en particulier dans les tentatives de découpage en tranches que l'on retrouve notamment dans les travaux de l'urbaniste autrichien Rudolf Giffinger, et qui sont pour l'essentiel orientés vers la justification d'une tentative de classification des « Smart cities » européennes. C'est ainsi qu'il propose de décomposer la «Ville intelligente» en six tranches : "Smart economy », "smart mobility », "smart governance », "smart people», «smart environment», «smart living». Fondée sur une batterie de critères extrêmement nombreux, la méthode de R. Giffinger permet en principe de mesurer l'intelligence et donc d'établir une classification entre les villes et territoires. Tout fonctionne comme si l'intelligence avait été clairement définie pour se prêter à ce jeu de décomposition et de mesure... Ce qui est évidemment contestable.

Il est ici intéressant de se rappeler qu'un engouement semblable s'était cristallisé autour du concept de " classe créative » proposé en 2002 par Richard Florida. Le concept était lui aussi découpé en « tranches », ce qui permettait d'envisager son opérationnalisation. Pour attirer des « classes créatives », il convenait pour une collectivité de privilégier tolérance et diversité au sein de sa population. Et de proposer des instruments de mesure au travers de trois indicateurs : l'indicateur « bohémien », l'indicateur « gay » et l'indicateur forgé sur le pourcentage de personnes nées à l'étranger. C'étaient ces trois réunis qui 
produisaient l'indicateur composite de diversité (Composite Diversity Index - CDI ${ }^{12}$.

\section{Un défaut de problématisation politique}

Dans l'enchaînement récent des politiques publiques, notamment sur la scène internationale, l'expression « Smart City » renvoie à une tentative d'accorder les enjeux du développement d'une « société de l'information», qui se décline en « société du savoir» puis en « économie de la connaissance » et de « développement durable». Lors du Sommet Habitat III de Quito, la « Ville intelligente » correspond bien à cette tentative, comme le souligne très clairement l'article $66, \mathrm{du}$ « Nouveau Programme pour les Villes » adopté à l'issue de l'événement : "Nous nous engageons à adopter le concept de ville intelligente, qui tire parti des perspectives ouvertes par la numérisation, les énergies et les technologies propres ainsi que les transports innovants, offrant ainsi aux habitants les moyens d'opérer des choix plus respectueux de l'environnement et de favoriser une croissance économique durable, et aux villes la possibilité d'améliorer leur offre de services $^{13}$."

Or, à la différence de ce qui s'est passé autour des concepts sur lesquels il s'appuie, celui de «Ville intelligente » apparaît comme dénué de ces fondations.

Les concepts de «développement durable » comme celui de "société de l'information » sont contemporains dans leur accession au statut d'enjeux politiques et dans leur mise sur agenda des grandes institutions nationales et internationales. On est alors entre les décennies
1960 et 1970 . C'est ce parcours d'accession que nous proposons d'appeler la « problématisation politique ». Cette problématisation correspond à une construction politique qui, à partir d'une ou d'un ensemble d'idées, aboutit à son adoption par les acteurs politiques puis sa mise sur agenda. Cette problématisation correspond à une prise de conscience de l'existence d'enjeux traduisibles en enjeux politique. Elle suit un cheminement complexe parce qu'il n'est pas linéaire ni logique, et qu'il transite par une série d'étapes spécifiques.

Ces idées ont rapidement besoin d'incarnations pour progresser. D’idées, elles deviennent questions, enjeux... Leurs premières incarnations sont les individus qui se retrouvent à l'interface des mondes politiques, médiatiques, associatifs, scientifiques. Ils ont en quelques sortes un rôle de passeurs, ils acquièrent le statut du héraut, du porte-voix des enjeux en question. Dans l'accès à l'agenda national français, le rapport Nora/ Minc sur « L'informatisation de la société » est un document d'autant plus intéressant qu'il explicite très clairement quelles sont ses principales références : «(...), ce rapport ne serait pas ce qu'il est si nous n'avions pu nous appuyer sur des travaux antérieurs, traitant de l'informatique et de la société. Il s'agit notamment de ceux de MM. Maurice Allègre, Jacques Attali, Michel Crozier, Robert Lattès, Jacques Lesourne, Pierre Lhermitte, Bruno Lussato, Edgar Morin, Joël de Rosnay, François Sarda, Bertrand Schwartz, Michel Serres, Alain Touraine, Bernard Tricot $»^{14}$

On y retrouve des acteurs, considérés comme des pionniers de l'informatique : Maurice Allègre qui a été le Délégué à l'Informatique du Plan Calcul 
et donc un acteur central d'un programme qui est l'ancêtre de celui que réclament les auteurs du rapport, Pierre Lhermitte, président fondateur du CIGREF (Club Informatique des Grandes Entreprises Françaises) en 1970 qui voisine avec des hauts fonctionnaires (Bertrand Schwartz, Bernard Tricot), des sociologues (Michel Crozier, Edgar Morin, Alain Touraine), un philosophe (Michel Serres), des économistes (Jacques Lesourne, Jacques Attali), un mathématicien (Robert Lattès). Plusieurs personnes de cette courte liste permettent d'incarner la figure du «passeur» dans la mesure où, dans leur carrière comme dans leur position sociale, ils articulent fréquemment le monde de la pensée ou de la recherche et celui de l'action. Jacques Attali, de même que Jacques Lesourne ou Joël de Rosnay s'inscrivent bien dans cette logique. Ils sont parmi les auteurs qui organisent un transfert entre ces «mondes». D'une certaine manière, on peut interpréter la « carrière politique » des idées qu'ils exposent à l'aune de leur carrière personnelle, entre le monde académique, celui d'essayiste, et celui de conseiller politique.

Côté politiques environnementales, le document qui fait en quelque sorte le pendant du rapport Nora/Minc mais au niveau international est le rapport au Club de Rome de 1972. Ce rapport, réalisé par une équipe du MIT et dirigé par Dennis Meadows, reposait sur une simulation par ordinateur du fonctionnement du système planétaire. «Ce sont les travaux des économistes écologiques Kenneth E. Boulding (1966) et Herman E. Daly (1971) qui se trouvent en arrière-plan de ce premier rapport au Club de Rome et de la perspective d'un "état d'équilibre durable" qui est mise en avant. ${ }^{15}$ » Le message politique qui est exprimé dans ce rapport propose d'envisager un acte politique particulièrement fort, qui adhère à l'idée qu'il y a des « Limits to Growth ». C'est dans le cadre de la Conférence des Nations Unies sur l'environnement humain, organisé à Stockholm en 1972 que ce « message » transfuse vers l'organisation onusienne et donne corps au concept politique d' " écodéveloppement ». L'expression d'écodéveloppement apparaît alors comme un choix diplomatique et stratégique, porté par le Secrétaire Général de la Conférence afin de ne pas condamner les politiques développementalistes revendiquées par les pays du Sud en proposant des limites au développement des pays du Nord.

Le concept, ou la notion, voire le slogan ou la marque que représente l'expression « Smart city » n'a pas bénéficié, en prélude à son apparition, de tels phénomènes de circulations entre mondes politiques, monde des experts, des médias et monde académique. C'est notamment ce qui explique la réticence de nombreux représentants du monde scientifique à son égard.

Si on en reste au cas français, le travail parlementaire visible au travers des rapports, nombreux et généralement de très bonne qualité, est un bon indicateur de l'interpénétration entre les différents mondes qui participent à la problématisation politique. Or, alors même que plusieurs villes, en fait plutôt des métropoles, se revendiquent ouvertement des « Villes intelligentes », le premier, et à ce jour le seul rapport officiel qui porte sur ces questions est celui réalisé en avril 2017 par le député Luc Belot : « De la smart city au territoire d'intelligence $(\mathrm{s})^{16} »$, Rapport au Premier ministre sur l'avenir de la smart city, La Documentation 
française, avril 2017.

\section{Les principaux constituants des «Villes in- telligentes »}

Au-delà de leur forte hétérogénéité, de leur charge politique, de leur instrumentalisation politique, on peut toutefois observer quelques traits communs entre la plupart des modèles en débat. Nous proposons d'en retenir trois principaux : le recours et l'exploitation des mégadonnées, la modernisation voire la transformation des services urbains et l'affichage d'une volonté politique de s'appuyer sur la participation des habitants.

Le principe même, le modèle économique des mégadonnées (big data en anglais) consiste à trouver de l'utilité à ces énormes quantités d'informations produites par les activités urbaines. Pour les acteurs publics urbains, l'intérêt premier de cette ressource est de permettre une amélioration du gouvernement de la ville. Pour les acteurs économiques, il s'agit de mettre sur le marché de nouveaux services urbains, le plus souvent accessibles sur des plateformes numériques. Dans de nombreuses « Villes intelligentes », on trouve des partenariats incluant l'acteur public (la ville ou la métropole), des grandes entreprises de services urbains et des « start-uppers » au sein de structures qui leur sont parfois dédiées (le programme Data City du Numa à Paris, ou encore le Tuba à la Métropole de Lyon).

La « Ville intelligente » est une ville qui a optimisé son fonctionnement dans toute une série de directions, en particulier en matière de production ou de distribution de services urbains adaptés aux besoins traditionnels comme aux besoins ou attentes nouveaux de la population et ceci dans la perspective du développement durable. C'est ainsi que les secteurs de l'eau, de l'assainissement, de l'énergie, des transports publics, des télécommunications... sont au cœur de nombreux projets de villes intelligentes. À Medellin par exemple, le bâtiment de l'EPM (Empresas Públicas de Medellín), qui est une entreprise prestataire de services publics en matière de gestion déléguée des services de distribution d'énergie, de gaz, d'eau et de télécommunications fondée en 1955, s'appelle tout simplement « immeuble intelligent $»$. Au sein de la politique municipale qui consiste à développer une "Ciudad Inteligente », EPM est un opérateur fondamental. La politique municipale de la «Ciudad intelligente » est peut-être avant tout traduite dans les innovations de toutes natures introduites par l'EPM dans la production et la distribution des services sur un espace urbain de près de 2,4 millions d'habitants. L' « immeuble intelligent » est aussi le lieu de stockage et d'exploitation d'une partie des mégadonnées produites par la ville.

Enfin la question de la «participation des habitants » est sans doute l'une de celle qui est considérée par la plupart des acteurs comme à la fois l'une des plus nécessaires et l'une des plus compliquées. En un sens, la première participation que puisse attendre un acteur public urbain, c'est que sa ville, intelligente ou non, soit habitée. Or, dans quelques-uns des grands modèles très connus (celui de Songdo notamment, mais on pourrait aussi citer Masdar et quelques autres), ce qui semble manquer le plus, ce sont les habitants.

Faire place, de façon très explicite, à la partici- 
pation des habitants est d'autant plus important pour les promoteurs des «Villes intelligentes » que le fait d'émettre cette proposition leur permet d'anticiper sur toute une série de contestations pouvant provenir de la société civile comme des habitants. Dans de nombreux cas, il s'agit de montrer que ce qui prime, c'est moins la technologie que l'humain. Dans de nombreux cas, au travers de la ville intelligente, il s'agit aussi de faire droit à la montée en puissance d'un habitant qui, par le recours aux technologies numériques, devient aussi et de plus en plus un acteur de la ville. Plusieurs expériences en cours dans des « laboratoires urbains » rendent compte de ce phénomène. Ces « laboratoires urbains » (souvent appelés Urban Labs, même en France quand ils existent) s'efforcent d'organiser une meilleure diffusion de l'information municipale en direction de la population en passant par le « recrutement » d'habitants appelés à participer à des phases de tests de services ou d'actions dans l'espace public. Ces participations peuvent d'ailleurs aller jusqu'à des engagements budgétaires, en particulier dans le cadre des actions menées autour des « budgets participatifs ».

Ce triptyque : recours aux mégadonnées, modernisation/transformation des services urbains et participation des habitants ne se retrouve évidemment pas à l'identique dans l'ensemble des projets de « Villes intelligentes ». En outre, il apparaît clairement que certains sont plus orientés vers la mise en œuvre de projets urbains que l'on pourrait qualifier de radicalement nouveaux, s'apparentant en cela à des utopies urbaines (on n'en trouve pas en France, mais en Chine, en Inde, à Dubaï...), d'autres étant plutôt conçus autour de performances technologiques (se réclamant du principe de « solutionnisme technologique »), comme à Singapour, Santander... tandis que d'autres se préoccupent prioritairement de « développement durable » (c'est en particulier le cas de la plupart des cas des villes d'Europe septentrionale).

On peut donc essayer d'en conclure, provisoirement, que les villes intelligentes sont à la croisée de leur histoire, qu'il n'est pas du tout certain qu'elles ne se scindent pas en plusieurs sousgroupes, mais que, en même temps, il ne semble pas, pour le moment tout du moins, qu'une autre expression, aussi synthétique et attractive, puisse aujourd'hui s'imposer. À défaut, il convient sans doute de considérer que les «Villes intelligentes » font désormais partie des questions pertinentes dans la recherche urbaine. 


\section{$R \cdot E ́ F \cdot E ́ R \cdot E \cdot N \cdot C \cdot E \cdot S$}

François ASCHER, La ville hypermoderne ou Ces événements nous dépassent, feignons d'en être les organisateurs, Éd. de l'Aube, 2001.

Alain BOURDIN et Ariella MASBOUNGUI (dir.), Un urbanisme des modes de vie, Éd. Le Moniteur, 2004.

Antoine COURMONT, « Open data et recomposition du gouvernement urbain : de la donnée comme instrument à la donnée comme enjeu politique », in Informations sociales, 2015/5, $\mathrm{n}^{\circ}$ 191, p. 40-50.

Renata Paola DAMERI, « Searching for Smart City definition : a comprehensive proposal $»$, in International Journal of Computers \& Technology, Volume 11, Issue 5, 2013.

Rudolf GIFFINGER, Christian FERTNER, Hans KRAMAR, Robert KALASEK, Nataša PICHLER-MILANOVIĆ, Evert MEIJERS, Smart cities - Ranking of European medium sized cities, Center of Regional Science, Vienna UT, October 2007.

Raphaël LANGUILLON-AUSSEL, Nicolas LEPRÊTRE et Benoit GRANIER, « La stratégie de la « smart city » au Japon : expérimentations nationales et circulations globales », in EchoGéo, 36/2016 : avril 2016/ juin 2016.

Albert MEIJER, Manuel Pedro RODRÍGUEZ BOLÍVAR, « La gouvernance des villes intelligentes. Analyse de la littérature sur la gouvernance urbaine intelligente $»$, in Revue Internationale des Sciences Administratives, 2016/2 (Vol. 82), p. 417-435.

Antoine PICON, Smart Cities. Théorie et critique d'un idéal auto-réalisateur, Éditions B2, 2015. Pascal SOKOLOFF, « Le Big data et l'Open data au service des collectivités », in Annales des Mines - Responsabilité et environnement 2016/4, $n^{\circ} 84$, p. 41-46.

Anthony M. TOWNSEND, Smart Cities, Big Data, Civic Hackers, and the Quest for a New Utopia, W. W. Norton \& Company, 2014. 
$\mathrm{N} \cdot \mathrm{O} \cdot \mathrm{T} \cdot \mathrm{E} \cdot \mathrm{S}$

1. Dans la suite du texte, nous préférerons utiliser l'expression en langue française.

2. WOESSNER Raymond, «La territorialisation : proposition pour la compréhension du phénomène par une entrée systémique », in Revue d'Économie Régionale \& Urbaine, 2010/4, octobre, p. 675.

3. BERMOND Guillaume, «Boom des événements Smart Cities en Europe, Conférences, congrès et symposiums se multiplient en Europe de l'Ouest mais aussi de l'Est cette année », in Le Monde du 30 mars 2017. 4. IDC.

5. http://www.lagazettedescommunes.com/505137/ les-strategies-smart-city-gagnent-en-maturite/.

6. TACTIS, Appropriation et déploiement de la Smart City dans les villes, agglomérations, et territoires français, Observatoire Smart City, édition 2006, p. 45. 7. DATAR, Atlas international des technopoles, 1991. 8. PICON Antoine, Smart Cities, Éditions B2, Collection Actualités, p. 7.

9. Cf. par exemple le Guide « Smart City versus Stupid Village », réalisé en 2016 par la Caisse des Dépôts, l'association des Communautés de France et l'association des Petites Villes de France.

10. http://www.caissedesdepots.fr/smart-city-versusstupid-village.

11. Cf. ESTÈBE Philippe, L'égalité des territoires, une passion française, Éd. Puf, Collection la Ville en débat, 2015.

12. Cf. FLORIDA Richard, Cities and the Creative Class. New York, London : Routledge, 2005 ; The Rise of the Creative Class and How It's Transforming Work, Leisure and Everyday Life. New York : Basic Books, 2002.

13. ONU, Nouveau Programme Pour les Villes, Habitat III, Quito, 17-20 octobre 2016, p. 22.
14. NORA Simon et MINC Alain, L'informatisation de la société, rapport au président de la République, La Documentation française, 1978, Annexe, p. 134.

15. VIVIEN Franck-Dominique, «Les antécédents conceptuels du développement soutenable »; in B. Zuindeau (éd.); Développement durable et territoire, Éd. Septentrion, 1010, p. 26.

16. BELOT Luc, De la smart city au territoire d'intelligence(s), Rapport au Premier ministre sur l'avenir de la smart city, La Documentation française, avril 2017. 


\section{$R \cdot E ́ \cdot S \cdot U \cdot M \cdot E ́$}

Les «Villes intelligentes » sont encore assez largement des objets curieux autant que de curiosité au sein des sciences sociales. Elles ont du mal à s'imposer comme des objets d'étude parce qu'elles ne constituent pas une catégorie facile à identifier et à objectiver. Une des raisons principales de cette difficulté tient au fait que, en même temps que ce sont de proto-objets scientifiques, ce sont aussi des produits commerciaux. Rien là de très inhabituel, c'est souvent le cas des questions nouvelles lorsqu'elles cherchent à s'imposer et avant que ne se mette en place le processus de circulation des idées entre les différents acteurs impliqués soit directement soit indirectement dans la conception des politiques publiques. Nous ne proposons pas ici de définition scientifique de la « Ville intelligente », nous nous efforçons de mettre en lumière à quelles questions scientifiques renvoie cette émergence et ce en quoi elle propose d'éventuelles réponses. Peu importe que l'expression «Ville intelligente » s'implante ou non, elle cristallise pour le moment une part significative des questions émergentes dans le développement urbain contemporain : la place des mégadonnées, la modernisation/transformation" des services urbains, le statut d'acteurs-discrets des habitants/citoyens...

\begin{abstract}
"Smart cities" are still largely curious objects as much as curiosity in the social sciences. They have difficulty in establishing themselves as objects of study because they are not an easy category to identify and objectify. One of the main reasons for this difficulty is that, along with scientific proto-objects, they are also commercial products. This is not unusual: it is often the case with new questions when they are trying to impose themselves and before the process of circulation of ideas
\end{abstract}

between the different actors involved either directly or indirectly in the design of public policies. We do not suggest here a scientific definition of the "smart city", we try to highlight to what scientific questions this emergence refers and in what it suggests possible answers. Regardless of whether or not the term "smart city" is being implemented, it is currently capturing a significant share of emerging issues in contemporary urban development: the place of big data, the modernization / transformation of urban services, the status of discrete actors of the inhabitants / citizens ... 
